\title{
Reading Assistance for Blind
}

\author{
Adithya Asokan', Afsal N S ${ }^{2}$, Aswathy Nandakumar ${ }^{3}$, Aswathy Rajan ${ }^{4}$, Sherin Rappai ${ }^{5}$, Rachana M K \\ UG Scholar, Electronics and Communication, IES College of Engineering, Thrissur, India ${ }^{1,2,3,4,5}$
}

Asst. Professor, Electronics and Communication, IES College of Engineering, Thrissur, India ${ }^{6}$

\begin{abstract}
A Majority of the visually impaired use Braille for reading documents and books which are difficult to make and less readily available. This gives rise to the need for the development of devices that could bring relief to the agonizing tasks that the visually impaired has to go through. Due to digitization of books there are many excellent attempts at building a robust document analysis system in industries, academia and research labs, but this is only for those who are able to see. This project aims to study the image recognition technology with speech synthesis and to develop a cost effective. The project has a small inbuilt camera that scans the text printed on a paper, converts it to audio format using a synthesized voice for reading out the scanned text quickly translating books, documents and other materials for daily living, especially away from home or office. Not only does this save time and energy, but also makes life better for the visually impaired as it increases their independency.
\end{abstract}

Keywords: OCR, TTS, SRAM, EEPROM.

\section{INTRODUCTION}

Visual impairment, also known as vision impairment or vision loss, is a decreased ability to see to a degree that causes problems not fixable by usual means, such as glasses. Some also include those who have a decreased ability to see because they do not have access to glasses or contact lenses.

Braille is a tactile writing system used by people who are blind or visually impaired and it's costly. By making a device which can serve by translating the text to speech, we can reduce the complexity and expenses. We can avoid the complex conversion, i.e. is text to braille cells.

A key fact by WHO, 285 million people are estimated to be visually impaired worldwide: 39 million are blind and 246 have low vision. With all the various problems faced by blind people, the problem of reading is our field of interest. We are advancing in technology and science, but in spite of such development the techniques used by the blind are old fashioned. Most of the reading materials available for the blind are in the form of Braille.

A person has to learn using Braille just for reading, and if a person is unable to learn Braille then he will be unable to read. Another disadvantage is that an error in understanding will result in reading wrong data. The last disadvantage is that the books, documents etc. have to be converted into a form of raised dots for the blind to read. The books and papers available for the blind in Braille format are quite less in comparison to the vast pool of books which are printed daily. Hence a device to help the blind in reading is a necessity. This document provides details about using ATmega328 as the main unit which has an inbuilt camera that is used to scan any written document and uses Optical character recognition (OCR) to convert the image into a digital text.

We then use a text to audio system (text-to-speech converter) that will enable us to convert the digital text into a synthesized voice. The scanned image undergoes image processing for obtaining the region of interest and segmentation of the letters from the word. The segmented letters undergo OCR. The output is combined to obtain the individual words as it was present originally in the document.

The words obtained are given to a text to speech convertor that allows us to obtain the voice converted output according to the written document. As this system is less costlier, less complex and more effective the blind people can use this system without difficulty.

Thus our aim is to introduce an effective assistance for the visually impaired people in the form of a specially designed book.

\section{BLOCK DIAGRAM}

The block diagram explains about two sections, software section and hardware section. 


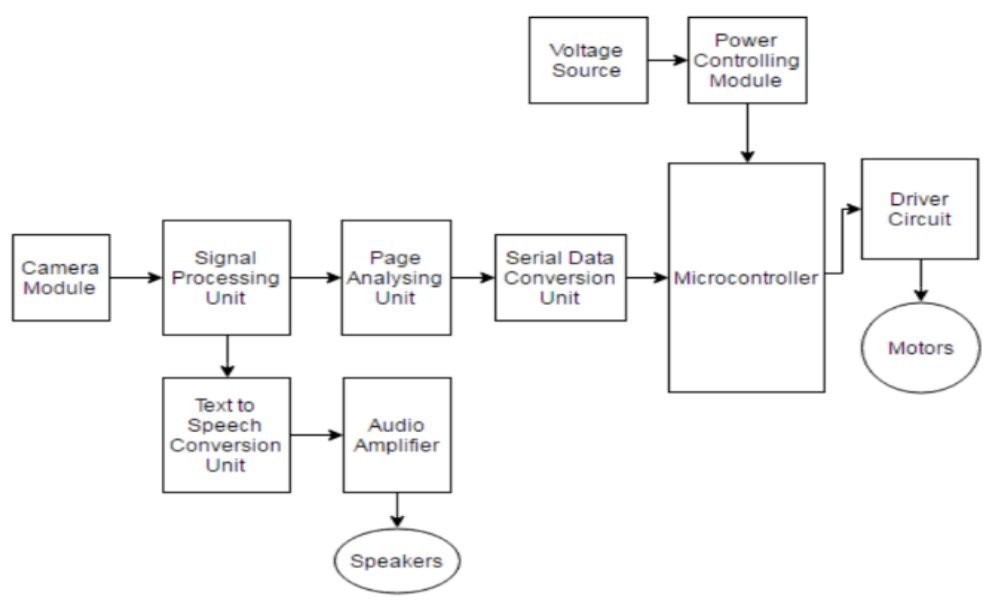

Fig. 1 Block diagram of proposed system

\section{III.BLOCK DESCRIPTION}

\section{A. ATMEGA328}

The ATmega48PA/88PA/168PA/328P provides the following features: 4K/8K bytes of In-System Programmable Flash with Read-While-Write capabilities, 256/512/512/1 K bytes EEPROM,512/1K/1K/2K bytes SRAM, 23 general purpose I/O lines, 32 general purpose working registers, three flexible Timer/Counters with compare modes, internal and external interrupts, a serial programmable USART, a byte-oriented 2-wire Serial Interface, an SPI serial port, a 6channel 10-bitADC (8 channels in TQFP and QFN/MLF packages), a programmable Watchdog Timer with internal Oscillator, and five software selectable power saving modes. The Idle mode stops the CPU while allowing the SRAM, Timer/Counters, USART, 2-wire Serial Interface, SPI port, and interrupt system to continue functioning. The Powerdown mode saves the register contents but freezes the Oscillator, disabling all other chip functions until the next interrupt or hardware reset. Power-save mode, the asynchronous timer continues to run, allowing the user to maintain a timer base while the rest of the device is sleeping. The ADC Noise Reduction mode stops the CPU and all I/O modules except asynchronous timer and ADC, to minimize switching noise during ADC convers4ions. In Standby mode, the crystal/resonator Oscillator is running while the rest of the device is sleeping. This allows very fast start-up combined with low power consumption. The device is manufactured using Atmel's high density non-volatile memory technology. The On-chip ISP Flash allows the program memory to be reprogrammed In-System through an SP serial interface, by a conventional non-volatile memory programmer, or by an on chip Boot program running on the AVR core.

\section{B. Servomotors}

Servo motors (or servos) are self-contained electric devices that rotate or push parts of a machine with great precision. Servos are found in many places: from toys to home electronics to cars and airplanes. If you have a radio-controlled model car, airplane, or helicopter, you are using at least a few servos. In a model car or aircraft, servos move levers back and forth to control steering or adjust wing surfaces. By rotating a shaft connected to the engine throttle, a servo regulates the speed of a fuel-powered car or aircraft. Servos also appear behind the scenes in devices we use every day. Electronic devices such as DVD and Blu-ray Disc TM players use servos to extend or retract the disc trays. In 21stcentury automobiles, servos manage the car's speed: The gas pedal, similar to the volume control on a radio, sends an electrical signal that tells the car's computer how far down it is pressed. The car's computer calculates that information and other data from other sensors and sends a signal to the servo attached to the throttle to adjust the engine speed. Commercial aircraft use servos and a related hydraulic technology to push and pull just about everything in the plane.

\section{Driver circuit}

In electronics, a driver is an electrical circuit or other electronic component used to control another circuit or component, such as a high-power transistor, liquid crystal display (LCD), and numerous others. They are usually used to regulate current flowing through a circuit or to control other factors such as other components, some devices in the circuit. The term is often used, for example, for a specialized integrated circuit that controls high-power switches in switched-mode power converters. An amplifier can also be considered a driver for loudspeakers, or a constant voltage circuit that keeps an attached component operating within a broad range of input voltages. Typically the driver stage(s) of a circuit requires different characteristics to other circuit stages. For example in a transistor power amplifier, typically the driver circuit requires current gain, often the ability to discharge the following transistor bases rapidly, and low output impedance to avoid or minimize distortion. 


\section{Speaker}

Speakers are one of the most common output devices used with computer systems. Some speakers are designed to work specifically with computers, while others can be hooked up to any type of sound system. Regardless of their design, the purpose of speakers is to produce audio output that can be heard by the listener. Speakers are transducers that convert electromagnetic waves into sound waves. The speakers receive audio input from a device such as a computer or an audio receiver

\section{E. Camera module}

Camera module is an image sensor integrated with control electronics and an interface like CSI, Ethernet or plain raw low voltage differential sinaling Camera module is only used to capture the image for signal processing unit. This can take high definition video as well as stills photograph.

F. Voltage source

Voltage source is a two terminal device which can maintain a fixed voltage. An ideal voltage source can maintain the fixed voltage independent of the load resistance or the output current. However, a real-world voltage source cannot supply unlimited current. A voltage source is the dual of a current source. Real-world sources of electrical energy, such as batteries, generators, and power systems, can be modelled for analysis purposes as a combination of an ideal voltage source and additional combinations of impedance elements

G. Text to speech converter

Speech synthesis is the artificial production of human speech. A computer system used for this purpose is called a speech synthesizer, and can be implemented in software or hardware. Text-to-speech is the generation of synthesized speech from text. A text-to-speech (TTS) system converts normal language text into speech; other systems render symbolic linguistic representations like phonetic transcriptions into speech. Synthesized speech can be created by concatenating pieces of recorded speech that are stored in a database. Systems differ in the size of the stored speech units; a system that stores phones or diaphones' provides the largest output range, but may lack clarity. For specific usage domains, the storage of entire words or sentences allows for high-quality output. Alternatively, a synthesizer can incorporate a model of the vocal tract and other human voice characteristics to create a completely synthetic voice output.

H. OCR

Optical character recognition (optical character reader, OCR) is the mechanical or electronic conversion of images of typed, handwritten or printed text into machine-encoded text, whether from a scanned document, a photo of a document, a scene-photo (for example the text on signs and billboards in a landscape photo) or from subtitle text superimposed on an image (for example from a television broadcast).[1] It is widely used as a form of information entry from printed paper data records, whether passport documents, invoices, bank statements, computerised receipts, business cards, mail, printouts of static-data, or any suitable documentation. It is a common method of digitising printed texts so that they can be electronically edited, searched, stored more compactly, displayed on-line, and used in machine processes such as cognitive computing, machine translation, (extracted) text-to-speech, key data and text mining. OCR is a field of research in pattern recognition, artificial intelligence and computer vision.

\section{IV.RESULT}

Using matlab version 2015, we executed the program for text to speech conversion. After completely converting the image copied by the first camera, then the second camera proceeds scanning and produces the texts converted in to speech and are amplified in to audio signals. These signals are then given to a speaker. A page analysing unit is used to analyse corresponding pages. Three servo motors are used in order to turn the pages. The three servo motors are, (a) Roller servo: for rolling the pages (b) Lift servo: for lifting the pages and (c) Stopper servo: for placing the lifted pages at the correct place.

\section{ADVANTAGES}

This system is simple in use and it can be easily used by the blind. If the people are illiterate, they can also use this. Common people can use the system in case, if they are busy with their works.

\section{VI.DISADVANTAGES}

The Cost of installation is high and it need separate space.

\section{VII.APPLICATIONS}

- Language translation.

- Algorithm to handle non horizontal text strings. 


\section{IJIREEICE \\ Vol. 5, Issue 4, April 2017}

\section{CONCLUSION}

Here we have studied the image recognition technology with speech synthesis and developed a cost effective technique. We designed a device which can serve by translating texts to speech. The books and papers available for the blind in Braille format are quite less in comparison to the vast pool of books which are printed daily. Hence a device to help the blind in reading is a necessity. The 'Reading Assistance for the blind' is not just a project that empowers the blind to become independent, but is also a resource saver. It cuts down the cost of printing Braille books along with the time and energy spent into doing so. This is a less costly solution to one of the many challenges that visually impaired face.

\section{ACKNOWLEDGMENT}

The authors would like to acknowledge the Management, Principal, Head of the department, all the faculties and staff of Electronics and Communication Department, of IES College of Engineering, Thrissur, Kerala, for their co-operation and technical guidance given during the entire course of the project work to complete it successfully. We would like to thank all our beloved classmates, friends and family for their encouragement.

\section{REFERENCES}

[1] Leija, L.; Santiago, S.; Alvarado, C. (31 Oct-3 Nov 1996 ), “A reading assistance for visually impaired person”, Bridging Disciplines for Biomedicine Proceedings of the 18th Annual International Conference of the IEEE, Vol. 1, no., pp.405-406 Vol. 1.

[2] Chucai Yi, Yingli Tian, Aries Arditi, "Portable camera-based assistive text and product label reading from hand-held objects for blind persons",IEEE/ASME Transactions on Mechatronics, vol. 19, issue. 3, June 2014, pp.302-312.

[3] Dhaval V. Waghulde, Kishor S. Gaikwad "Touchpad to read text document for blind people", SSRG International Journal of Electronics and Communication Engineering(SSRG-IJECE), vol. 2, issue. 11, November 2015.

[4] J. C Bliss, "A direct translation reading aid for the blind", Proceedings of the IEEE, vol. 54, issue. 1, January 2013, pp. 41-48.

[5] Bazzi, I.; Schwartz, R.; Makhoul, J. (Jun 1999), An Omni font open-vocabulary OCR system for English and Arabic, Pattern Analysis and Machine Intelligence, IEEE Transactions on vol.21, no.6, pp.495-504.

[6] V. Ajantha Devi, Dr. S Santhosh Baboo (Jul-Aug2014), Optical Character Recognition on Tamil Text Image Using Raspberry Pi International Journal of Computer Science Trends and Technology (IJCST) - Vol. 2 Issue 4

\section{BIOGRAPHIES}

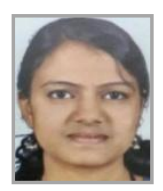

Adithya Asokan currently pursuing the Bachelor's degree in Electronics and Communication Engineering from the IES College of Engineering, Thrissur.

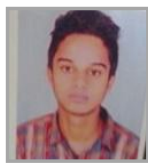

Afsal NScurrently pursuing he Bachelor's degree in Electronics and Communication Engineering from the IES College of Engineering, Thrissur.

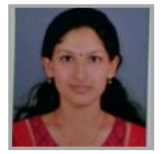

Aswathy nandakumar currently pursuing the Bachelor's degree in Electronics and Communication Engineering from the IES College of Engineering, Thrissur.

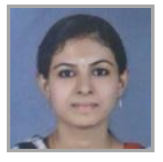

Aswathy rajancurrently pursuing the Bachelor's degree in Electronics and Communication Engineering from the IES College of Engineering, Thrissur.

Sherin rappaicurrently pursuing the Bachelor's degree in Electronics and Communication Engineering from the IES College of Engineering, Thrissur.

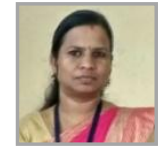

Rachana M K Acompleted Master's Degree in VLSI . She is currently working as Assistant Professor in Department of Electronics \& Communication Engineering, IES College of Engineering, Thrissur. 